11,10

\title{
Фазовый переход металл-диэлектрик в тонких пленках диоксида ванадия, легированного никелем
}

\author{
(C) В.Н. Андреев, В.А. Климов
}

Физико-технический институт им. А.Ф. Иофрфе РАН, Санкт-Петербург, Россия

E-mail: vn.andreev.solid@mail.ioffe.ru

Поступила в Редакцию 24 мая 2019 г.

В окончательной редакции 24 мая 2019 г.

Принята к публикации 27 мая 2019 г.

Исследована электропроводность тонких поликристаллических пленок $\mathrm{VNi}_{x} \mathrm{O}_{2}$ в широком интервале температур, охватывающем области как металлической, так и диэлектрической фазы. Показано, что с ростом концентрации никеля температура фазового перехода металл-диэлектрик понижается, а ширина температурной области сосуществования фаз монотонно увеличивается. Для объяснения температурной зависимости электропроводности диэлектрической фазы $\mathrm{VNi}_{x} \mathrm{O}_{2}$ применена модель прыжковой проводимости, учитывающая влияние тепловых колебаний атомов на резонансный интеграл. Вычислены значения параметра $\varepsilon$ в зависимости от степени легирования $\mathrm{VO}_{2}$.

Ключевые слова: фазовый переход, электропроводность, легирование, полярон.

DOI: 10.21883/FTT.2019.10.48272.491

\section{1. Введение}

В свободных от механических напряжений монокристаллах чистого диоксида ванадия $\left(\mathrm{VO}_{2}\right)$ при охлаждении ниже $T_{c}=340 \mathrm{~K}$ наблюдается структурный фазовый переход первого рода из металлической тетрагональной фазы $(R$-фаза) в диэлектрическую моноклинную фазу $\left(M_{1}\right.$-фаза $)[1,2]$. Этот переход сопровождается скачкообразным изменением электрических и оптических параметров кристаллических образцов. Практическое применение диоксида ванадия основано на вышеупомянутом скачке электрических и оптических констант, а также на том, что фазовое превращение совершается за очень короткое ( 100fs) время [3]. Фазовый переход носит мартенситный характер и сопровождается возникновением заметных упругих напряжений. Благодаря этому температурные зависимости оптических и электрических параметров имеют вид петли гистерезиса. Сильное влияние на эти зависимости оказывает введение иновалентных катионных примесей. Введение таких примесей замещения, как $\mathrm{Mo}^{6+}$ или $\mathrm{W}^{6+}$, являющихся донорами, приводит к образованию в кристаллической решетке диоксида ванадия состояния $\mathrm{V}^{3+}$ [4]. Легирование донорами сопровождается заметным понижением температуры фазового перехода $R \rightarrow M_{1}$ и постепенной его деградацией, то есть постепенным уменьшением „скачка“ электропроводности и отражательной способности, сопровождающего фазовый переход. Напротив, введение акцепторных примесей, таких как $\mathrm{Al}^{3+}$ или $\mathrm{Cr}^{3+}$, сопровождаемое заменой $\mathrm{V}^{4+}$ на $\mathrm{V}^{5+}$, не только увеличивает температуру фазового перехода, но изменяет сам характер его. Вместо диэлектрической фазы $M_{1}$ при охлаждении образуется диэлектрическая моноклин- ная фаза $M_{2}$, отличающаяся от первой тем, что в ней спарена лишь половина атомов ванадия, тогда как другая половина при фазовом переходе лишь смещается из центра кислородных октаэдров [5,6]. Следует отметить, что образование $M_{2}$-фазы не связано непосредственно с акцепторными свойствами трехвалентных примесей. Как показано в [7], фаза $M_{2}$ возникает и в нелегированных монокристаллах $\mathrm{VO}_{2}$ в случае приложения к ним одноосных механических напряжений. Ранее было показано, что всестороннее сжатие монокристаллов чистого диоксида ванадия приводит к повышению температуры фазового перехода. Все это подчеркивает важность подхода, при котором катионы примесей рассматриваются не просто как доноры или акцепторы, но и как центры внутреннего расширения или сжатия кристаллической структуры соответственно. Наличие внутренних или внешних упругих напряжений оказывает сильнейшее влияние на процесс перехода металлдиэлектрик (ПМД) в диоксиде ванадия [4-15]. В [6,15] сделан вывод о том, что процесс фазового перехода металл-диэлектрик в диоксиде ванадия контролируется этими упругими напряжениями.

Несмотря на то, что $\mathrm{VO}_{2}$ интенсивно исследуется на протяжении нескольких десятилетий, до сих пор не решен вопрос о природе ПМД в этом соединении. До конца не ясно, как влияют электрон-фононное взаимодействие и электронные корреляции на характеристики этого оксида. Исследованию легированных монокристаллов и тонких пленок диоксида ванадия в последние годы уделяется большое внимание, поскольку это может позволить объяснить природу фазового перехода [16-19]. Экспериментальные данные по электропроводности чистого и легированного диоксида 
ванадия, как правило, фрагментарны и проведены в узком температурном диапазоне. Ранее мы исследовали электропроводность чистого диоксида ванадия в широком интервале температур и показали, что электроперенос в $\mathrm{VO}_{2}$ осуществляется прыжками поляронов малого радиуса, на которые оказывают влияние тепловые колебания решетки [1]. В рамках такого подхода в предлагаемой работе мы рассматриваем свойства диоксида ванадия, легированного никелем. Ранее такая система не изучалась, но можно было ожидать, что влияние никеля, который в большинстве химических соединений двухвалентен, на электропроводность и температуру фазового перехода металл-диэлектрик будет аналогичным тому, которое оказывают другие примеси акцепторы.

\section{2. Эксперимент}

Тонкие пленки диоксида ванадия с примесью никеля были синтезированы методом одновременного лазерного напыления из мишеней металлического V (99.9\%) и металлического $\mathrm{Ni}(99.8 \%)$ в атмосфере кислорода при температуре 750-900 К. Так как температуры плавления ванадия и никеля близки, о степени легирования $(x)$ судили по относительному времени испарения каждой из мишеней. В качестве подложек использовали монокристаллический $\mathrm{Al}_{2} \mathrm{O}_{3}$ (0001). Толщина пленок составляла примерно $100 \mathrm{~nm}$.

Электропроводность синтезированных тонких пленок измерялась с помощью стандартной четырех зондовой методики. Перед началом синтеза пленок диоксида ванадия методом лазерного напыления в вакууме на подложку наносились платиновые электроды толщиной примерно $150 \mathrm{~nm}$. За процессом фазового перехода также следили по изменению отражательной способности исследованных пленок на длине волны $1.54 \mu \mathrm{m}$.

\section{3. Результаты и обсуждение}

Температурные зависимости электропроводности тонких пленок чистого и легированного никелем диоксида ванадия представлены на рис. 1. Видно, что в отличие от случая легирования $\mathrm{Al}^{3+}$ или $\mathrm{Cr}^{3+}$ введение примеси никеля сопровождается понижением температуры фазового перехода. Одновременно с увеличением степени легирования увеличивается и электропроводность $\sigma$ диэлектрической фазы оксида, а величина скачка электропроводности уменьшается. При этом уменьшение скачка $\sigma$ происходит не только за счет увеличения электропроводности диэлектрической фазы, но и за счет монотонного уменьшения электропроводности металлической фазы по мере увеличения концентрации легирующей примеси. Электропроводность металлической фазы чистого $\mathrm{VO}_{2}$ равна $\sigma_{m} \sim 10^{3} \Omega^{-1} \cdot \mathrm{cm}^{-1}$, что близко к моттовскому пределу для минимальной металлической проводимости [20], однако характер $\sigma(T)$ не является

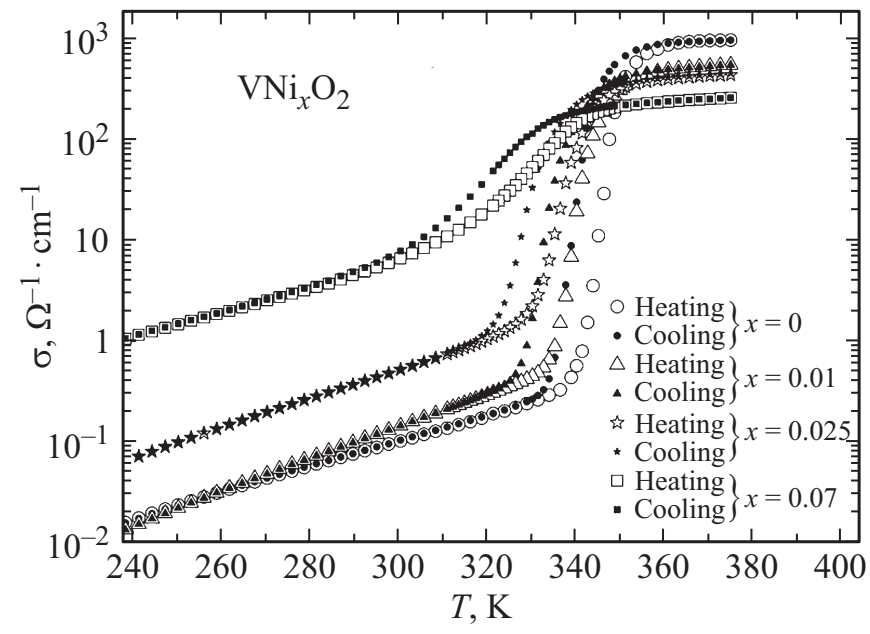

Рис. 1. Температурные зависимости электропроводности пленок $\mathrm{VNi}_{x} \mathrm{O}_{2}$ при изменении концентрации никеля.

металлическим. Это, по нашему мнению, связано с андерсоновской локализацией на дефектах и межзеренных границах (пленки поликристаллические). В монокристаллах $\sigma_{m} \sim 10^{4} \cdot \mathrm{cm}^{-1}$ и проводимость носит металлический характер [1]. По мере легирования $\mathrm{VO}_{2}$ никелем электропроводность металлической фазы постепенно снижается до $\sigma_{m} \sim 10^{2} \Omega^{-1} \cdot \mathrm{cm}^{-1}$. Подобное уменьшение электропроводности металлической фазы ранее мы наблюдали в тонких пленках диоксида ванадия, легированных водородом [21], который не является примесью замещения. Если рассматривать никель как примесь замещения, то в символике Крёгера-Винка растворение $\mathrm{NiO}$ в диоксиде ванадия при условии, что оно сопровождается образованием только электронных дефектов, может быть записано следующим образом:

$$
\mathrm{NiO} \rightarrow \mathrm{Ni}_{\mathrm{V}}^{\prime \prime}+2 h+\frac{1}{2} \mathrm{O}_{2} \uparrow,
$$

то есть растворение молекулы окиси никеля сопровождается появлением в решетке диоксида ванадия двух дырок $(h)$. Это в свою очередь может повлечь за собой образование двух ионов $\mathrm{V}^{5+}$, которые являются внутренними центрами сжатия кристаллической структуры. Таким образом, если реализуется реакция (1), можно ожидать, как и в случае легирования хромом и алюминием, появления фазы $M_{2}$ и повышения температуры фазового перехода. В действительности ни того, ни другого мы не наблюдали.

Для объяснения полученного результата можно предположить, что примесь никеля не является примесью замещения, а входит в междоузлия структуры диоксида ванадия. Тогда реакция растворения $\mathrm{NiO}$ в $\mathrm{VO}_{2}$ может быть представлена так

$$
\mathrm{NiO} \rightarrow \mathrm{Ni}_{i}^{x}+\frac{1}{2} \mathrm{O}_{2} \uparrow
$$


Ионизация междоузельного атома никеля будет приводить к тому, что он станет донором

$$
\mathrm{Ni}_{i}^{x} \rightarrow \mathrm{Ni}_{i}^{\bullet}+e
$$

Если согласно реакции (2) вхождение никеля в междоузлия действительно имеет место, то диоксид ванадия перестает быть стехиометричным и будет содержать избыток ионов металла. Избыток металла в окислах приводит к результату, совершенно аналогичному случаю с недостатком кислорода, а в диоксиде ванадия это вызывает понижение температуры фазового перехода, которое мы и наблюдаем для исследованных образцов на рис. 1. В этом случае формула легированного никелем диоксида ванадия должна быть записана как $\mathrm{VNi}_{x} \mathrm{O}_{2}$. Атом никеля, внедренный в междоузлие, уже сам по себе является центром внутреннего расширения. Ионизация междоузельных атомов никеля приведет к возникновению ионов $\mathrm{V}^{3+}$. Такие ионы также можно рассматривать, как внутренние центры упругого расширения кристаллической структуры диоксида ванадия. Наличие таких центров понижает $T_{c}$. Ключевым моментом образования диэлектрической фазы является спаривание соседних ионов ванадия, то есть образование ковалентной связи $\mathrm{V}^{4+}-\mathrm{V}^{4+}$. При замене в такой паре одного из ионов $\mathrm{V}^{4+}$ ионом $\mathrm{V}^{3+}$ энергия связи будет уменьшаться, поскольку „лишний“ электрон расположится на антисвязывающей молекулярной орбитали. Уменьшение энергетического выигрыша при ПМД также потребует большего переохлаждения и приведет к понижению температуры фазового перехода.

На рис. 2, $a, b$ соответственно показаны зависимости $\sigma(T)$ в области ПМД при нагреве и охлаждении тонких пленок чистого $\mathrm{VO}_{2}$ и $\mathrm{VNi}_{x} \mathrm{O}_{2}(x=0.01,0.025$ и 0.07). Температурная область ПМД в легированных тонких пленках уширяется с ростом концентрации никеля на фоне уменьшающегося скачка электропроводности $\Delta \sigma$. Уширение температурной области сосуществования фаз может быть вызвано постепенным превращением фазового перехода первого рода в переход второго рода из-за размытия фазовой границы металл-диэлектрик.

Результаты измерения электропроводности чистого и легированного никелем $\mathrm{VO}_{2}$ при охлаждении до температуры $T=240 \mathrm{~K}$ показаны на рис. 1. Видно, что зависимость $\sigma(T)$ в диэлектрической фазе линейна в координатах $\lg (\sigma)$ от $T$. Ранее мы исследовали электропроводность диоксида ванадия и показали, что его проводимость описывается прыжками поляронов малого радиуca, на которые влияют тепловые колебания решетки [1]. Так как механизм переноса заряда в диэлектрической фазе при легировании диоксида ванадия меняться не должен, то уместно рассматривать электропроводность $\mathrm{VNi}_{x} \mathrm{O}_{2}$ в рамках модели Брыксина [22]. В этой модели учитывается эффект теплового смещения атомов решетки на вероятность междоузельных перескоков малых поляронов. Смещения атомов приводят к изменениям в
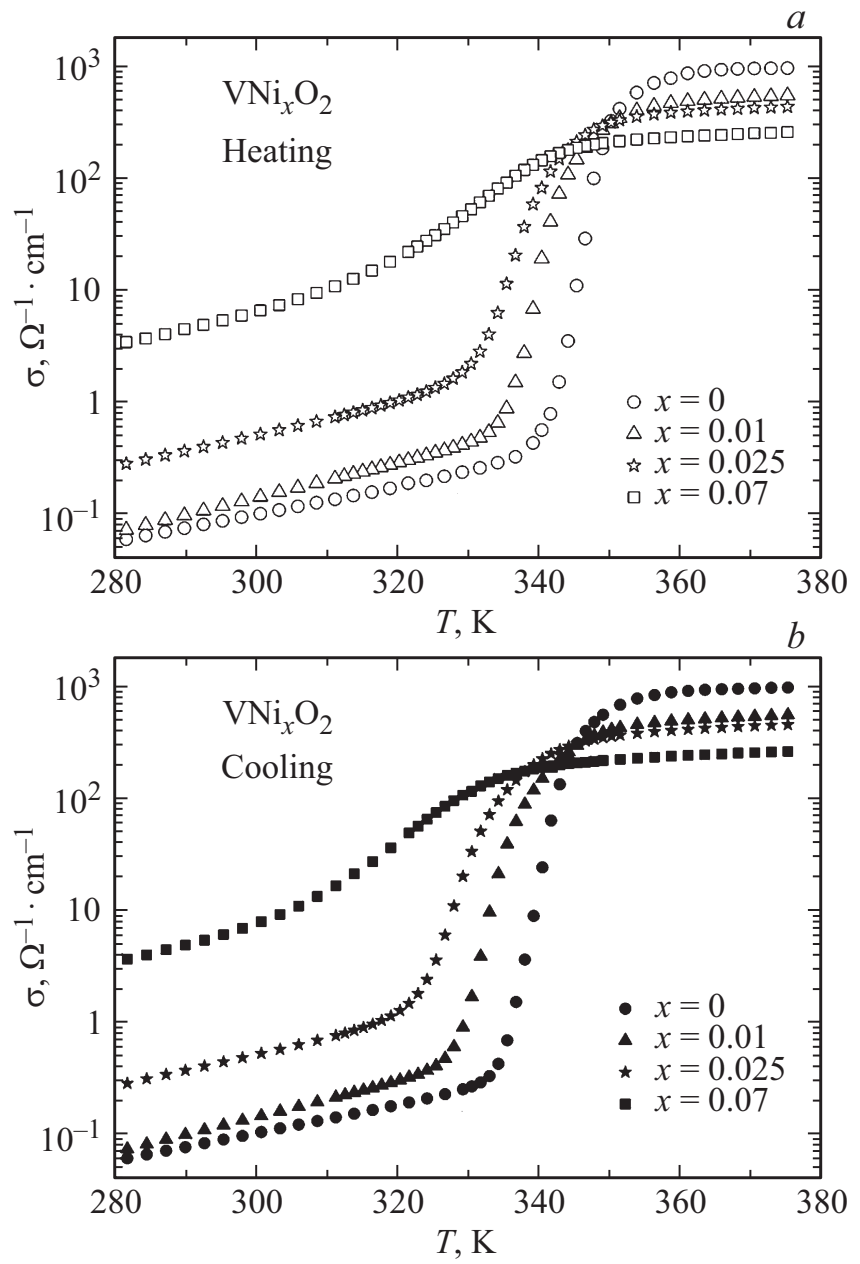

Рис. 2. Температурные зависимости электропроводности пленок чистого и легированного никелем $\mathrm{VO}_{2}$ в области фазового перехода. $a-$ полученные при нагревании; $b-$ полученные при охлаждении.

перекрытии волновых функций состояний на соседних узлах. Этим перекрытием определяется резонансный интеграл $(I)$. В первом приближении $I$ меняется с расстоянием $(R)$, на котором происходит перескок, как $\exp (-\alpha R)$, где $\alpha^{-1}$ есть эффективный радиус локализации. В свою очередь, прыжковая подвижность носителя заряда, определяющая электропроводность диоксида ванадия пропорциональна $I^{2}$. Для малых величин $\alpha^{-1}$, порядка амплитуды решеточных колебаний $(\rho)$, можно предположить, что $I^{2}$ должен зависеть линейно от $\rho$. Поэтому в случае зависимости $I^{2}$ от $\rho$ допустимо заменить $I^{2}$ на $\left\langle I^{2}\right\rangle$, где угловые скобки обозначают фононное усреднение через перенормировку фактора Дебая-Уоллера.

$$
\left\langle I^{2}\right\rangle=I^{2} \exp \left(2 \alpha^{2}\left\langle\rho^{2}\right\rangle\right)
$$

где $\left\langle\rho^{2}\right\rangle$ - среднеквадратичное тепловое смещение атомов на узлах решетки. Расчет прыжковой проводимости в модели поляронов малого радиуса с учетом влияния тепловых колебаний решетки на резонансный интеграл 


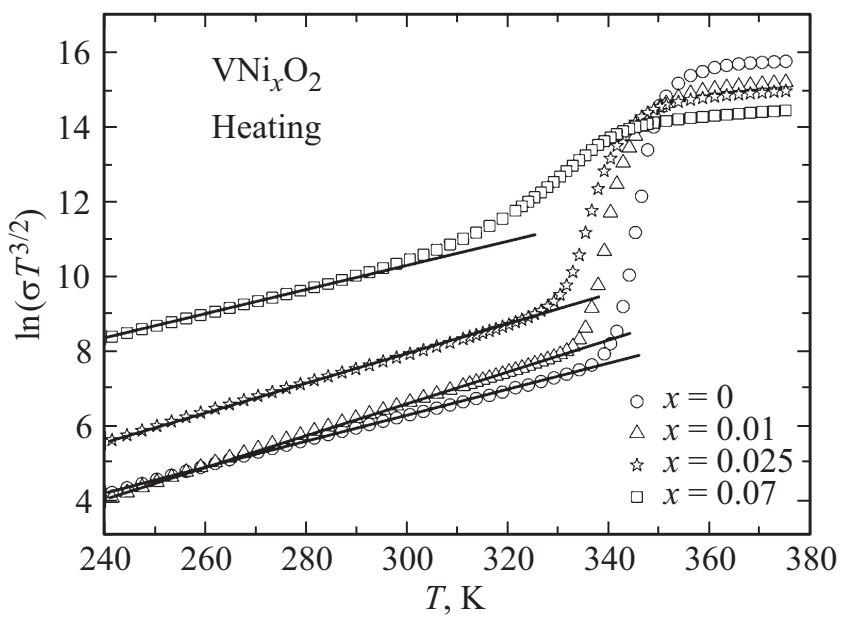

Рис. 3. Зависимость $\ln \left(\sigma T^{3 / 2}\right)=A+k_{B} T / \varepsilon$ пленок $\mathrm{VNi}_{x} \mathrm{O}_{2}$ с разной концентрацией никеля.

приводит к следующей зависимости электропроводности от температуры

$$
\sigma=e n \frac{e a^{2}}{2 h} \frac{\pi^{1 / 2} I^{2}}{E_{a}^{1 / 2}\left(k_{B} T\right)^{3 / 2}} \exp \left\{-E_{a} / k_{B} T+k_{B} T / \varepsilon\right\},
$$

где $a-$ постоянная решетки, $E_{a}-$ энергия, требуемая для осуществления перескока электрона, $n-$ концентрация носителей заряда, $e-$ заряд электрона, $h$ - постоянная Планка, $\varepsilon-$ величина, имеющая размерность энергии и учитывающая влияние тепловых колебаний решетки на резонансный интеграл. В области высоких температур, когда $2 k_{B} T>\hbar \omega_{q}$ (где $\hbar=h / 2 \pi$, $\omega_{q}$ - частота оптического фонона), $\varepsilon$ - связана со среднеквадратичным тепловым смещением $\left\langle\rho^{2}\right\rangle$ соотношением

$$
\varepsilon=k_{B} T / 2 \alpha^{2}\left\langle\rho^{2}\right\rangle .
$$

Анализ выражения (4) показывает, что при низких температурах второй член под экспонентой становится пренебрежимо малым по сравнению с первым, тогда как при высоких температурах доминирует уже второй член. Поэтому в низкотемпературном пределе выражение (4) может быть представлено в виде

$$
\ln \left(\sigma T^{3 / 2}\right)=A-E_{a} / k_{B} T,
$$

где $A$ и $E_{a}$ не зависят от температуры.

Напротив, в высокотемпературном пределе выражение (4) может быть представлено как

$$
\ln \left(\sigma T^{3 / 2}\right)=A+k_{B} T / \varepsilon,
$$

где $A$ и $\varepsilon$ не зависят от температуры.

В [1] мы показали, что $\sigma(T) \mathrm{VO}_{2}$ в области температур выше $T \sim 240 \mathrm{~K}$ может быть описана зависимостью (7). Эти зависимости для чистого $\mathrm{VO}_{2}$ и $\mathrm{VNi}_{x} \mathrm{O}_{2}$ $(x=0.01,0.025$ и 0.07$)$ представлена на рис. 3. Из сравнения чистого $\mathrm{VO}_{2}$ и $\mathrm{VNi}_{x} \mathrm{O}_{2}$ с относительно малым содержанием никеля $(x=0.01)$ видно, что наклон прямой,равный $\varepsilon^{-1}$, достаточно сильно увеличивается по сравнению с наклоном в чистом $\mathrm{VO}_{2}$. Значения $\varepsilon$ для $\mathrm{VO}_{2}$ и $\mathrm{VNi}_{x} \mathrm{O}_{2}(x=0.01)$ получились равными $2.48 \cdot 10^{-3} \mathrm{eV}, 2.025 \cdot 10^{-3} \mathrm{eV}$ соответственно. При дальнейшем увеличении концентрации никеля параметр $\varepsilon$ растет и в случае $\mathrm{VNi}_{x} \mathrm{O}_{2}(x=0.025$ и 0.07$)$ величина $\varepsilon$ получилась равной $2.155 \cdot 10^{-3} \mathrm{eV}, 2.69 \cdot 10^{-3} \mathrm{eV}$ соответственно. Из (3) и (5) следует, что величина $\varepsilon$ пропорциональна времени туннелирования малого полярона через барьер между соседними узлами. Иными словами подвижность полярона тем больше, чем меньше величина $\varepsilon$. Из наших данных следует, что при малых концентрациях никеля в диоксиде ванадия $\varepsilon$ уменьшается, а это можно трактовать как уменьшение локализации носителя заряда на узле. Однако, при дальнейшем увеличении концентрации никеля в $\mathrm{VNi}_{x} \mathrm{O}_{2}$ параметр $\varepsilon$ растет, что связано с увеличением локализации носителя заряда на узле.

\section{4. Заключение}

Показано, что легирование никелем диоксида ванадия приводит к существенному изменению температурной зависимости электропроводности $\mathrm{VNi}_{x} \mathrm{O}_{2}$ по сравнению с чистым $\mathrm{VO}_{2}$. Из полученных результатов сделан вывод о том, что никель в диоксиде ванадия находится в междоузлиях. Высказано предположение о том, что дополнительное размытие области ПМД при увеличении степени легирования тонких пленок $\mathrm{VO}_{2}$, может быть следствием размытия фазовой границы $R \rightarrow M_{1}$. Электропроводность диэлектрической фазы $\mathrm{VNi}_{x} \mathrm{O}_{2}$ хорошо описывается моделью малого полярона, учитывающей влияние тепловых колебаний атомов решетки на резонансный интеграл. Определен характерный параметр модели $\varepsilon$ для чистого и легированного никелем $\mathrm{VO}_{2}$. Показано, что энергия $\varepsilon$, при легировании уменьшается по сравнению с чистым $\mathrm{VO}_{2}$, но затем по мере роста концентрации Ni $\varepsilon$ непрерывно увеличивается. Это, по-видимому, связано с конкуренцией процесса увеличения параметров решетки и механизмов спаривания ионов ванадия при легировании.

\section{Финансирование работы}

Работа частично поддержана научной программой Президиума РАН.

\section{Конфликт интересов}

Авторы заявляют об отсутствии конфликта интересов.

\section{Список литературы}

[1] В.Н. Андреев, В.А. Климов. ФТТ 49, 2146 (2007).

[2] A. Zilbersztejn, N.F. Mott. Phys. Rev. B 11, 4383 (1975).

[3] A. Cavalleri, Cs. Toth, C.W. Siders, J.A. Squier, F. Raksi, P. Forget, J.C. Kiefer. Phys. Rev. Lett. 87, 237401-1(2001). 
[4] В.Н. Андреев, В.А. Климов. ФТТ 61, 1519 (2019).

[5] В.Н. Андреев, В.А. Климов, М.Е. Компан, Б.А.Мелех. ФТТ 56, 1802 (2014)

[6] A. Tselev, I.A. Luk'yanchuk, I.N. Ivanov, J.D. Budai, J.Z. Tischler, E. Strelkov, K. Jones, R. Hrokscg, A. Kolmakov, S.V. Kalinin. Nano Lett. 10, 2003 (2010).

[7] M. Marezio, D.B. Mc Whan, J.P. Remeika, P.D. Dernier. Phys. Rev. B 5, 2541 (1972).

[8] J. Wu, Q. Gu, B.S. Guiton, N.P. de Leon, L. Ouyang, H. Park. Nano Lett. 6, 2313 (2006).

[9] H. Park, J.M. Coy, T.S. Kasirga, C. Huang, Z. Fei, S. Hunter, D.H. Gobden. Nature 500, 431 (2013).

[10] В.Н. Андреев, В.А. Климов, М.Е. Компан. ФТТ 55, 1982 (2013).

[11] В.Н. Андреев, В.А. Климов, М.Е. Компан. Письма в ЖТФ 39, 12, 57 (2013)

[12] В.Н. Андреев, В.А. Климов. ФТТ 58, 590 (2016).

[13] В.Н. Андреев, В.А. Климов. ФТТ 53, 538 (2011).

[14] E. Strelkov, A. Tselev, I. Ivanov, J.D. Budai, J. Zhang, J.Z. Tischler, I. Kravchenko, S.V. Kalinin, A. Kolmakov. Nano Lett. 12, 6198 (2012).

[15] A. Tselev, E. Strelkov, I.A. Luk'yanchuk, J.D. Budai, J.Z. Tischler, I.N. Ivanov, K. Jones, A. Kolmakov, S.V. Kalinin. Nano Lett. 10, 4409 (2010).

[16] T.J. Hanlon, J.A. Coath, M.A. Richardson. Thin Solid Films 436, 269 (2003).

[17] C. Marini, E. Arcangeletti, D. Di Castro, L. Baldassare, A. Perucchi, S. Lupi, L. Malavasi, L. Boeri, E. Pomjakushina, K. Conder, P. Postorino. Phys. Rev. B 77, 235111 (2008).

[18] В.Н. Андреев, В.А. Климов, М.Е. Компан, Б.А. Мелех. ФТТ 56, 1802 (2014).

[19] В.Н. Андреев, В.А. Климов. ФТТ 60, 2425 (2018).

[20] N.F. Mott. Metall-Insulator Transitions. Tailor and Francis, LTD, London (1974).

[21] В.Н. Андреев, В.А. Климов, М.Е. Компан. ФТТ 54, 562 (2012).

[22] В.В. Брыксин. ЖЭТФ 100, 1556 (1991).

Редактор Т.Н. Василевская 